\title{
Temperature Compensation of Complex Permittivities of Biological Tissues and Organs in Quasi-Millimeter-Wave and Millimeter-Wave Bands
}

\author{
Taiji Sakai $^{1} \cdot$ Kanako Wake $^{1} \cdot$ Soichi Watanabe ${ }^{1} \cdot$ Osamu Hashimoto $^{2}$
}

\begin{abstract}
This study proposes a temperature compensation method of the complex permittivities of biological tissues and organs. The method is based on the temperature dependence of the Debye model of water, which has been thoroughly investigated. This method was applied to measured data at room temperature for whole blood, kidney cortex, bile, liver, and heart muscle. It is shown that our method can compensate for the Cole-Cole model using measured data at $20{ }^{\circ} \mathrm{C}$, given the Cole-Cole model based on measured data at $35{ }^{\circ} \mathrm{C}$, with a root-mean-squared deviation of $3 \sim 11$ $\%$ and $2 \sim 6 \%$ for the real and imaginary parts of the complex permittivities, respectively, among the measured tissues.

Key words : Biological Tissues, Complex Relative Permittivities, Temperature Compensation.
\end{abstract}

\section{Introduction}

Complex permittivities of biological tissues and organs are indispensable parameters for developing biomedical applications of electromagnetic fields (EMFs) and assessing the EMF safety of wireless communication techniques. The most widely used complex permittivity database was developed by Gabriel et al. [1 4], who measured the complex permittivities of several dozens of tissues and organs from $10 \mathrm{~Hz}$ to $20 \mathrm{GHz}$. Their Cole-Cole model has been used for determining complex permittivities up to $100 \mathrm{GHz}$.

In the quasi-millimeter wave and millimeter wave frequency region, the dielectric polarization of water molecules is dominant and known as $\gamma$-dispersion [5]. The relaxation frequency of the water molecules is around $20 \mathrm{GHz}$, the upper limit frequency of Gabriel's measurements. This suggests the possible limitation of the ColeCole models based on Gabriel's measurement data up to $20 \mathrm{GHz}$.

To enhance knowledge of the complex permittivities of biological tissues and organs in quasi-millimeter wave and millimeter-wave bands, we have recently conducted comprehensive measurements of the complex permittivities of biological tissues and organs [6].

Our measurements were conducted using biological samples at room temperature. Because the complex permittivities of biological tissues depend on temperature, it is necessary to correct the measured complex permittivi- ties to $36{ }^{\circ} \mathrm{C}$. In this paper, therefore, we propose a compensation method for the complex permittivities of biological tissues and organs in quasi-millimeter wave and millimeter wave bands.

\section{Methods and Materials}

\section{2-1 Measurement System}

The measurement system used in this study consists of a coaxial probe (Agilent Technology 85070E, Performance Probe) and a network analyzer (Agilent Technology E8316A). The coaxial probe, Performance Probe, is a newer type, while the slim-type coaxial probe, called Slim Form Probe, has been used for millimeter-wave measurements. The Performance Probe shows an improvement given its temperature characteristics: the available temperature range is -40 to $200{ }^{\circ} \mathrm{C}$, compared with 0 to $125{ }^{\circ} \mathrm{C}$ for the other probe. The difference between the Performance Probe and Slim Form Probe is described in Appendix.

The typical accuracy of the measurement system is specified as $5 \%$ [7]. The uncertainty of the measurement system with the slim-type probe has been reported previously [8], and is largely applicable to this study.

\section{2-2 Tissue Samples}

Biological tissues and organs measured in this study

Manuscript received October 1, 2010 ; revised December 6, 2010. (ID No. 20101001-07J)

${ }^{1}$ National Institute of Information and Communications Technology, Tokyo, Japan.

${ }^{2}$ Department of Electrical Engineering and Electronics of Aoyama, Gakuin University, Kanagawa, Japan.

Corresponding Author : Taiji Sakai (e-mail : sake@nict.go.jp) 
were sampled from swine. The tissues are whole blood, kidney cortex, bile, liver and heart muscle. All of the tissues are of high-water-content while fat and bone are classified as low-water-content ones.

The samples were stored with minimum water-evaporation. The measurements of the complex permittivities were conducted within 12 hours of slaughter. The details of the preparation of the samples are described elsewhere [6].

\section{2-3 Measurement Conditions and Cole-Cole Parameters}

The temperature of the samples was regulated using a temperature-controlled water-bath (Fig. 1). The measurement temperature was set at $20{ }^{\circ} \mathrm{C}, 25{ }^{\circ} \mathrm{C}, 30{ }^{\circ} \mathrm{C}, 35^{\circ} \mathrm{C}$, and $40{ }^{\circ} \mathrm{C}$.

Measurement frequency is from $5 \mathrm{GHz}$ to $50 \mathrm{GHz}$. The $\gamma$-dispersion due to water-molecule dielectric polarization is dominant in this frequency region. We therefore develop Cole-Cole models with one relaxation term (Eq. 1) based on the measured data of the complex permittivities, while Gabriel et al. developed four-relaxation-terms Cole-Cole model, which have applicability from $10 \mathrm{~Hz}$ to $100 \mathrm{GHz}$ [3].

$$
\hat{\varepsilon}(\omega)=\varepsilon_{\infty}+\frac{\varepsilon_{s}-\varepsilon_{\infty}}{1+(j \omega \tau)^{(1-\alpha)}}+\frac{\sigma_{s}}{j \omega \varepsilon_{0}}
$$

The unknown parameters of the Cole-Cole model (Eq. 1), i.e. $\varepsilon_{\infty}, \varepsilon_{s}, \sigma_{d c}, \alpha$, and $\tau$, are determined with Levenberg-Marquardt method [9].

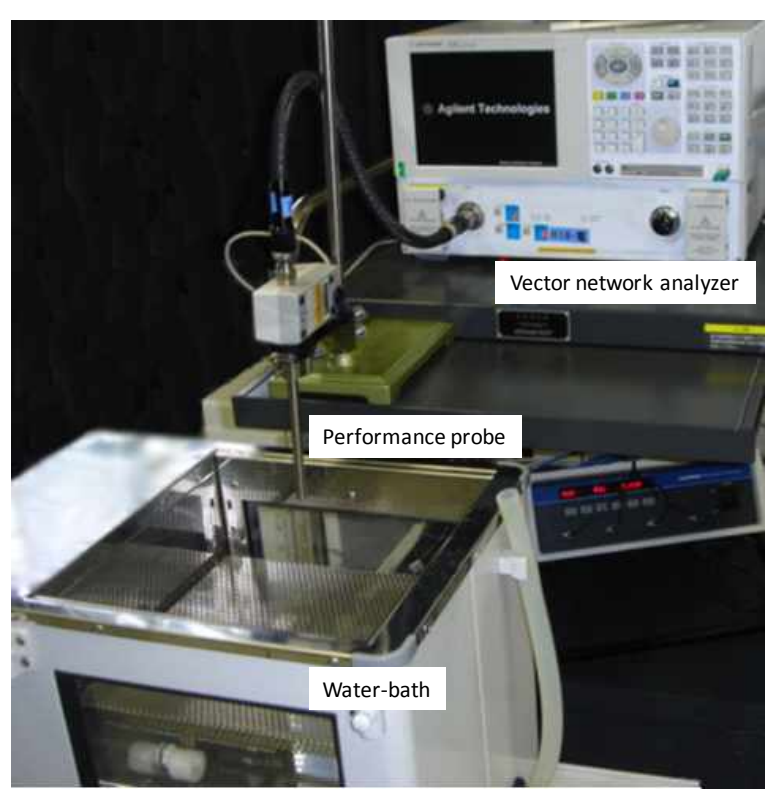

Fig. 1. A temperature-controlled water-bath for measurement of the complex permittivities of the biological tissues and organs.

\section{2-4 Temperature Compensation Method}

Because $\gamma$-dispersion is due to water-molecule dielectric polarization, we assumed that the temperature dependence of the complex permittivities of biological tissue and organs follows that of water in the frequency region where $\gamma$-dispersion is dominant, i.e., the quasi-millimeter wave and millimeter-wave frequency region.

The temperature dependence of the water has been thoroughly investigated [10]. Or and Wraith described the temperature dependence of the parameters of the Debye model of the water as follows:

$$
\begin{aligned}
\varepsilon^{*}(f) & =\varepsilon_{\infty}+\frac{\varepsilon_{s}-\varepsilon_{\infty}}{1+i\left(f / f_{\text {rel }}\right)}-i \frac{\sigma_{d c}}{2 \pi f_{\varepsilon_{0}}} \\
\varepsilon_{\infty}=4.25 & \\
\varepsilon_{s}(T)= & 87.74-0.4008 T+9.398 \times 10^{-4} T^{2} \\
& -1.410 \times 10^{-6} T^{3} \\
f_{\text {ref }}(T)= & \left(1.1109 \times 10^{-10}-3.824 \times 10^{-12} T\right. \\
& \left.+6.938 \times 10^{-14} T^{2}-5.096 \times 10^{-16} T^{3}\right)^{-1} \\
\sigma_{d c}(T)= & \sigma_{d c\left(25^{\circ} C\right)} \exp \left[-\Delta\left(2.033 \times 10^{-2}+1.266 \times 10^{-4} \Delta\right.\right. \\
& \left.\left.+2.464 \times 10^{-6} \Delta^{2}\right)\right] \\
& \approx \sigma_{d c\left(25^{\circ} C\right)}[1-0.02 \Delta] \\
\Delta=25 & -T
\end{aligned}
$$

Using the above temperature dependences, we assume that the temperature dependences of the Cole-Cole model parameters are as follows:

$$
\begin{aligned}
& \varepsilon_{\infty}(T)=\varepsilon_{\infty}\left(T_{m}\right)=\text { const. } \\
& \alpha(T)=\alpha\left(T_{m}\right)=\text { const. } \\
& \varepsilon_{s}(T)=\varepsilon_{s}\left(T_{m}\right) \cdot \varepsilon_{s, \text { water }}(T) / \varepsilon_{s, \text { water }}\left(T_{m}\right) \\
& f_{r e f}(T)=f_{r e f}\left(T_{m}\right) \cdot f_{r e f, \text { water }}(T) / f_{r e f, \text { water }}\left(T_{m}\right) \\
& \sigma_{d c}(T)=\sigma_{d c}\left(T_{m}\right) \cdot \sigma_{d c, \text { water }}(T) / \sigma_{d c, \text { water }}\left(T_{m}\right)
\end{aligned}
$$

where $T_{m}$ is the measurement temperature of the tissues and organs.

In this study, $T_{m}$ is $20^{\circ} \mathrm{C}$. We derived the Cole-Cole parameters at temperatures higher than $20{ }^{\circ} \mathrm{C}$ from (Eq. 4).

\section{Results}

Fig. 2 shows the frequency characteristics of the complex permittivities of the tissues and organs. The plotted data are from the Cole-Cole model based on measured data at each temperature and from the temperaturecompensated Cole-Cole model using (Eq. 4) with the Cole-Cole parameters based on measured data at $20{ }^{\circ} \mathrm{C}$.

It is shown that the complex permittivities significantly change with temperature. Generally good agree- 
ment is found for each tissue. To evaluate the deviation quantitatively, we show the deviation between the ColeCole model based on the measured data at each actual temperature and the Cole-Cole model at $20{ }^{\circ} \mathrm{C}$, compensated to each temperature (Fig. 3).

The root-mean-squared deviations between the ColeCole model at $35{ }^{\circ} \mathrm{C}$ and the temperature-compensated Cole-Cole model for all frequency regions are 3 11\% and $2 \sim 6 \%$ for the real and imaginary parts of the complex permittivities, respectively, among the measured tissues. The maximum deviations are $6 \sim 19 \%$ and $4 \sim 7$ $\%$ for the real and imaginary parts of the complex permittivities, respectively. A relatively large deviation appears at high frequencies for the real part of the complex permittivities especially with the heart muscle.

\section{Discussion}

We developed a temperature compensation method for the complex permittivities of the biological tissues and organs in the quasi-millimeter-wave and millimeter-wave frequency region. This method is based on the temperature dependence of the Debye parameters of water. We demonstrated that the compensated data agree with the actual-temperature data. A discussion of the method follows.

As shown in Fig. 3, relatively large deviations appear in the higher frequency region for the real part. The deviation at higher frequencies reflects the small value of the real part of the complex permittivities, where the relative error of the fitting Cole-Cole model increases with the fitting method used in this study [9]. The integrated absolute error is minimized.

We also discuss the impact of the EMF dosimetry between the compensated Cole-Cole model and the original Cole-Cole model. Fig. 4 shows the transmission coefficient, corresponding to the total power absorption of the biological body, a homogeneous slab (heart muscle), with normal TEM wave incidence from $5 \mathrm{GHz}$ to 50 $\mathrm{GHz}$ based on the complex permittivities of the compensated Cole-Cole model and the original Cole-Cole model.

No significant difference appears for all cases while a relatively large difference appears at higher temperatures and at higher frequencies; the maximum deviation is $3 \%$. This is interesting because the deviation of the real part of the complex permittivities significantly affect the power absorption by biological objects although

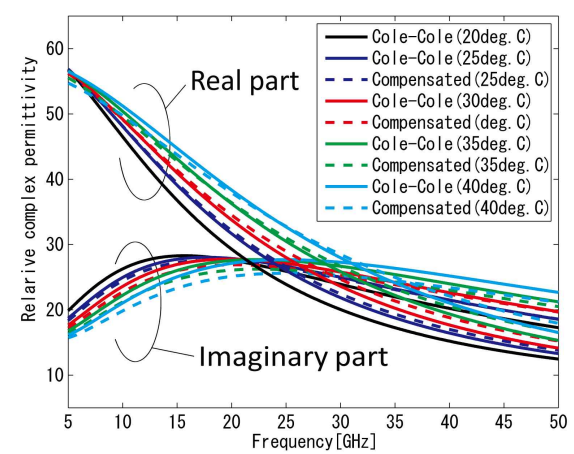

(a) Whole blood

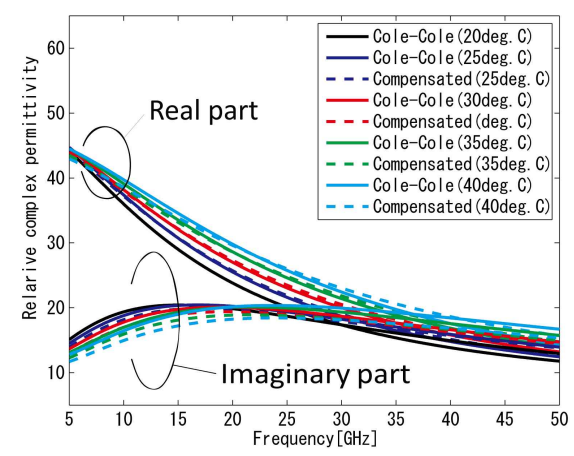

(d) Liver

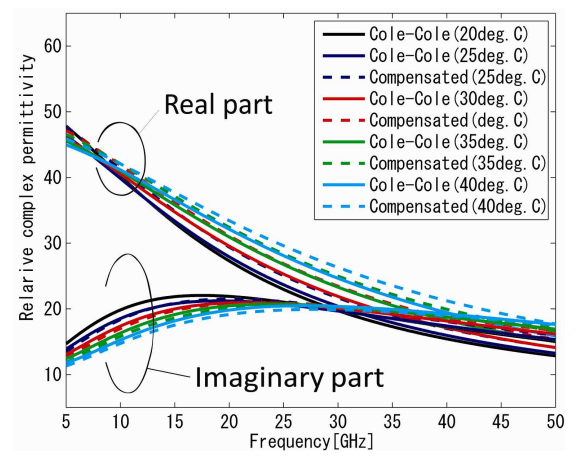

(b) Kidney cortex

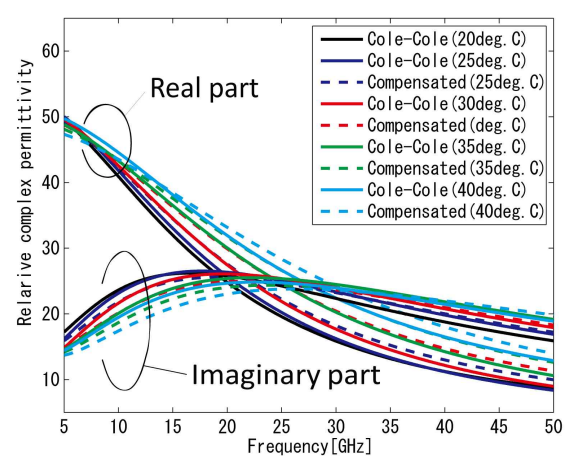

(e) Heart muscle

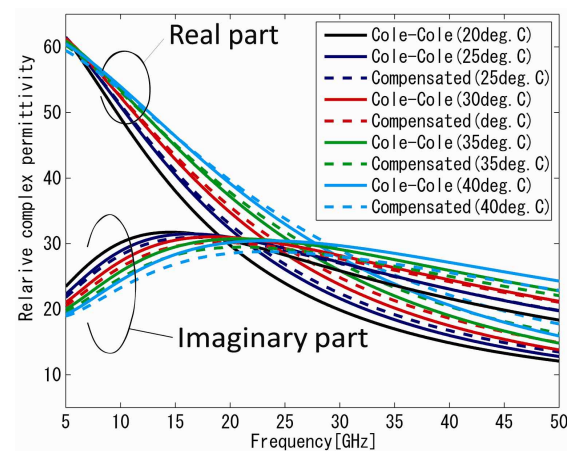

(c) Bile

Fig. 2. Frequency characteristics of the complex permittivities of the tissues and organs. The plotted data are from the Cole-Cole model based on measured data at each temperature and from the temperature-compensated Cole-Cole model using (Eq. 4) with the Cole-Cole parameters based on the measured data at $20{ }^{\circ} \mathrm{C}$. 


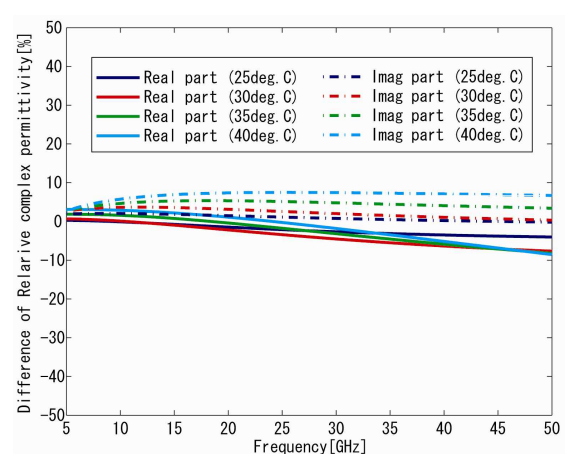

(a) Whole blood

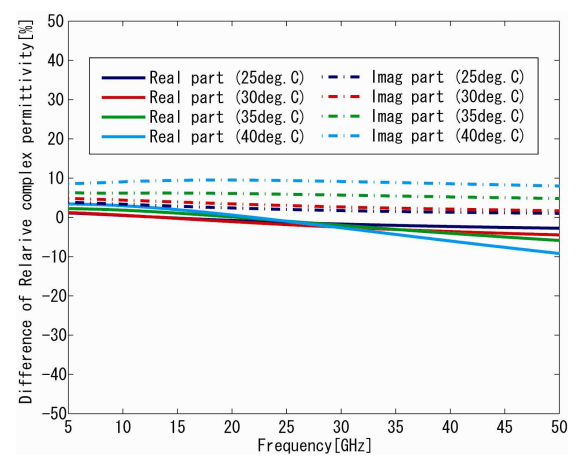

(d) Liver

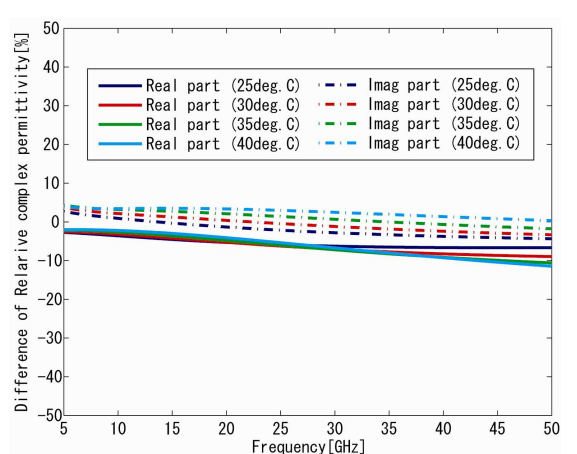

(b) Kidney cortex

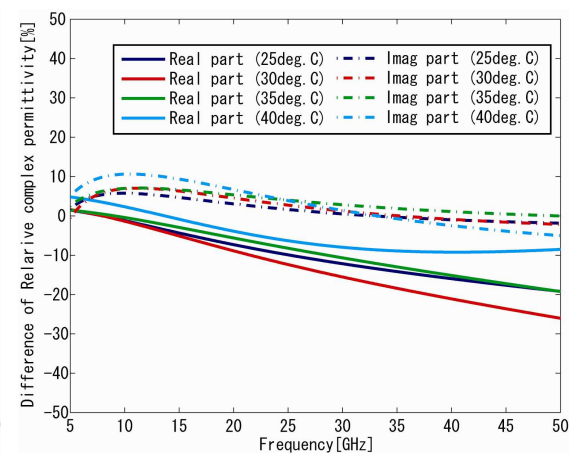

(e) Heart muscle

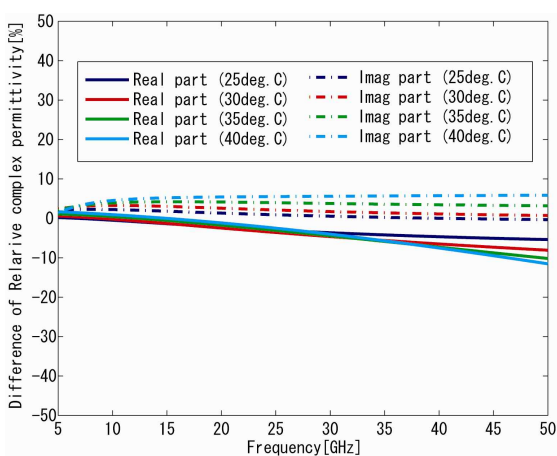

(c) Bile

Fig. 3. Deviation between the Cole-Cole model based on measured data at each actual temperature and the Cole-Cole model at $20{ }^{\circ} \mathrm{C}$, compensated to each temperature.

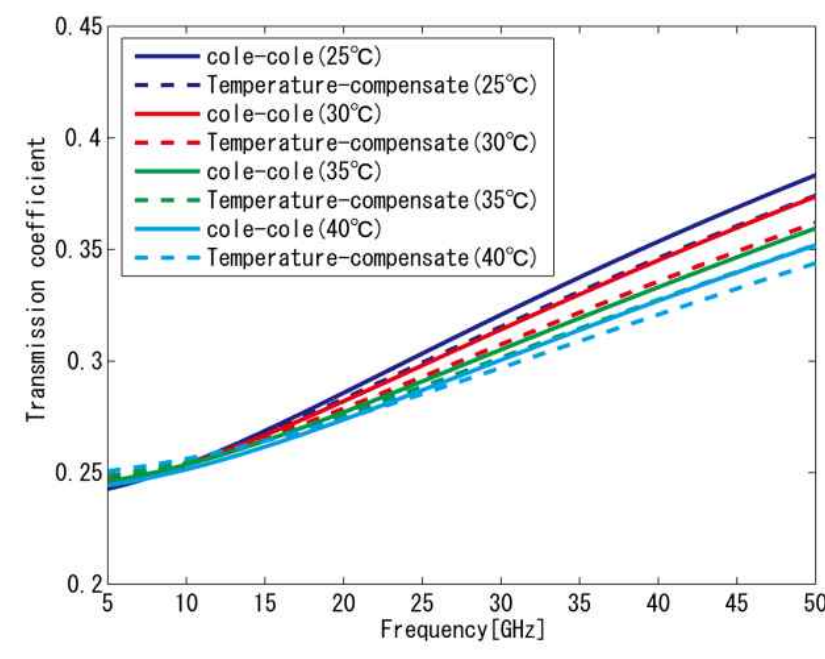

Fig. 4. Calculated transmission coefficients for TEM-wave normal incident with homogeneous slab of heart muscle. The complex permittivities are from the compensated Cole-Cole models or the original Cole-Cole models.

the imaginary part of the complex permittivities, i.e., conductivity, is more dominant for power absorption by biological objects in the lower frequency region, including cellular-phone operating frequency bands.
The temperature dependence of the complex permittivity of the biological tissues and organs has been investigated [11] although most previous studies focused on lower frequencies, i.e., up to several gigahertzes. Foster et al. reported that the temperature dependence is at most $2 \% /{ }^{\circ} \mathrm{C}$. Our results show reduced temperature dependence.

Finally we also note that Performance Probe is preferred for accurate measurement for temperatures higher than room temperature, or calibration temperature, as described in Appendix. However biological samples are frequently sampled in tiny quantities, e.g., a cornea. This may be a disadvantage for the Performance Probe because the probe requires a larger sample volume than the Slim Form Probe [7]. Our temperature-compensated method is therefore useful for cases in which the Slim Form Probe can only measure tiny biological samples.

\section{Conclusion}

We proposed a temperature compensation method of the complex permittivities of biological tissues and organs in the quasi-millimeter and millimeter wave frequency region. The method is based on the temperature dependence of the Cole-Cole parameters of water. 
We then compared the Cole-Cole model based on the measurement data at each actual temperature and the temperature-compensated Cole-Cole model based on the measurement data at room temperature. The comparison showed that our method can fairly approximate the temperature dependence of the complex permittivities from $5 \mathrm{GHz}$ to $50 \mathrm{GHz}$ with a root-mean-squared deviation of $3 \sim 11 \%$ and $2 \sim 6 \%$ for the real and imaginary parts of the complex permittivities, respectively, among the measured tissues.

Further investigation on other types of tissues and organs, especially tissues with low water content such as fat and bone, is necessary.

\section{Appendix}

A1. Comparison of complex permittivity measurements using Performance Probe and Slim Form Probe.

Fig. A1 shows the frequency characteristics of the complex permittivities of water at $36{ }^{\circ} \mathrm{C}$, higher than room temperature (i.e., nearly $22.5{ }^{\circ} \mathrm{C}$ ). The plotted data are derived from Cole-Cole models based on the measured data with Performance Probe and with Slim Form Probe. The calibration of both probe systems was done at room temperature.

The maximum deviations between the reference data [10] and the measured data with Performance Probe are $21 \%$ and $6 \%$ for the real and imaginary parts of the complex permittivities, respectively. The maximum deviations

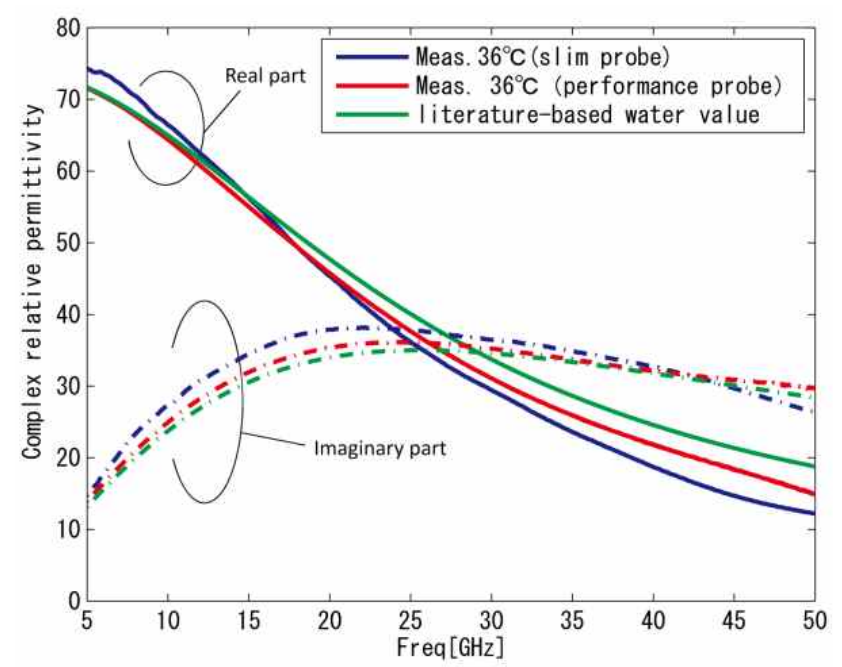

Fig. A1. Frequency characteristics of the complex permittivities of the water sample of Cole-Cole models based on measured data with the Performance Probe and with the Slim Form Probe. between the reference data and the measured data with Slim Form Probe are $35 \%$ and $17 \%$ for the real and imaginary parts of the complex permittivities. This suggests that Performance Probe is more appropriate for measurement at temperatures higher than room temperature.

\section{References}

[1] C. Gabriel, S. Gabriel, and E. Corthout, "The dielectric properties of biological tissues: I. Literature survey," Phys. Med. Biol., vol. 41, pp. 2231-2249, 1996.

[2] S. Gabriel, R. W. Lau, and C. Gabriel, "The dielectric properties of biological tissues: II. Measurements in the frequency range $10 \mathrm{~Hz}$ to $20 \mathrm{GHz}, "$ Phys. Med. Biol., vol. 41, pp. 2251-2269, 1996.

[3] S. Gabriel, R. W. Lau, and C. Gabriel, "The dielectric properties of biological tissues: III. Parametric models for the dielectric spectrum of tissues," Phys. Med. Biol., vol. 41, pp. 2271-2293, 1996.

[4] C. Gabriel, "Compilation of the dielectric properties of body tissues at RF and microwave frequencies," AL/OE-TR-1996-0037, Brooks Air Force Base, TX, USA, 1996.

[5] C. Gabriel, "Dielectric properties of biological materials," in Bioengineering and Biophysical Aspects of Electromagnetic Fields, edited by F. S. Barnes and B. Greenebaum, CRC Press, 2006.

[6] Sakai et al., "Measurement of complex permittivity and water contents of biological tissues in millimeter wave band," 32nd Annual Meeting of Bioelectromagnetics Society, Seoul, Korea, 2010.

[7] Agilent Technology, Agilent 85070E Dielectric Probe Kit $200 \mathrm{MHz}$ to $50 \mathrm{GHz}$ : Technical Overview, Agilent Technology 5989-0222EN, USA, 2008.

[8] Wakatsuchi, et al., "Reproducible measurements of complex permittivities of wet and solid biological tissues and organs from $500 \mathrm{MHz}$ to $50 \mathrm{GHz}$, IEEE, 2010.

[9] J. J. Moré, "The levenberg-marquardt algorithm: Implementation and theory," Numerical Analysis, Ed. G. A. Watson, Lecture Notes in Mathematics 630, Springer Verlag, pp. 105-116, 1977.

[10] D. Or, J. M. Wraith, "Temperature effects on soil bulk dielectric permittivity measured by time domain reflectometry: A physical model," Water Resources Research, vol. 35, pp. 371-383, 1999.

[11] K. R. Foster, H. P. Schwan, "Dielectric properties of tissues - a review," In C. Polk and E. Postow eds., Handbook of Biological Effects of Electromagnetic Radiation, CRC Press, Boca Raton, 1995. 


\section{Taiji Sakai}

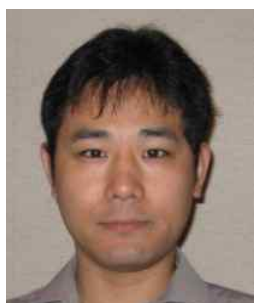

received the B.E., M.E., and Ph.D. degrees from Aoyama Gakuin University, Tokyo, Japan, in 2001, 2003, and 2006, respectively. He is currently an Expert Researcher with the Electromagnetic Compatibility (EMC) Group Applied Electromagnetic Research Center, National Institute of Information and Communications Technology (NICT), Tokyo Japan. He works for research on biomedical electromagnetic compatibility. $\mathrm{He}$ is a member of the Institute of Electronics, Information and Communication Engineers (IEICE), Japan, the Institute of Electrical and Electronics Engineering (IEEE). He received the 2005 Young Researcher Award from IEICE.

\section{Kanako Wake}

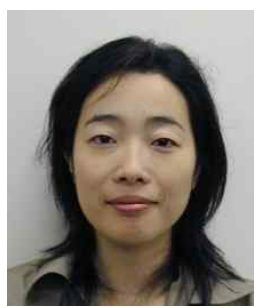

received the B.E., M.E., and D.E., degrees in electrical engineering from Tokyo Metropolitan University, Tokyo, Japan, in 1995, 1997 , and 2000, respectively. She is currently with the National Institute of Information and Communications Technology (NICT), Tokyo Japan. She works for research on biomedical electromagnetic compatibility. Dr. Wake is a member of the Institute of Electronics, Information and Communication Engineers (IEICE), Japan, the Institute of Electrical Engineers (IEE), Japan, the Institute of Electrical and Electronics Engineering (IEEE), and the Bioelectromagnetics Society. She received the 1999 International Scientific Radio Union (URSI) Young Scientist Award.

\section{Soichi Watanabe}

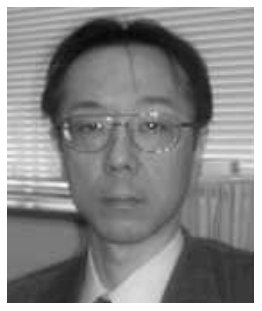

(Member, IEEE) received the B.E. M.E. and D.E. degrees in electrical engineering from Tokyo Metropolitan University, Tokyo, Japan, in 1991, 1993, and 1996, respectively. $\mathrm{He}$ is currently with the National Institute of Information and Communications Technology, Tokyo. His main research interest is biomedical electromagnetic compatibility. Dr. Watanabe is a member of the Institute of Electronics, Information and Communication Engineers (IEICE) of Japan, the Institute of Electrical Engineers (IEE), Japan, and the Bioelectromagnetics Society. He also has been a member of the Standing Committee on Physics and Engineering of International Commission on Non-Ionizing Radiation Protection since 2004. He received the 1996 Young Scientist Award from the International Scientific Radio Union, the 1997 Best Paper Award from IEICE, and the 2004 Best Paper Award (The Roberts Prize) from Physics in Medicine and Biology.

\section{Osamu Hashimoto}

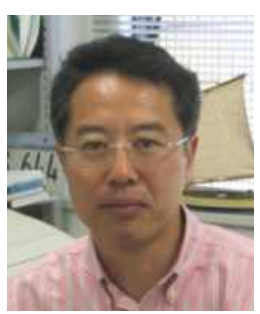

received his B.E. and M.E. in applied electronics engineering from the University of Electro-Communications, Japan in 1976 and 1978 and his doctorate from the Tokyo Institute of Technology, Japan, in 1986. He joined Toshiba Corporation in 1978. He joined the Defense Technical Development Laboratory in 1981. In 1991, he moved to the Department of Electrical Engineering and Electronics of Aoyama Gakuin University, Japan, as an associate professor, where he is currently a full professor. From 1994 to 1995 , he was with the University of Illinois as a guest researcher. He has been engaged in research on electromagnetic computation, microwave and millimeter-wave absorbers, planar filters, and measurement and analysis of radar crosssections. In 2006, he received the Electronics Society Award from the Institute of Electronics, Information, and Communication Engineers, and he was awarded the APMC 2006 Prize. $\mathrm{He}$ is the author/co-author of 28 books in Japanese including "Introduction to microwave absorbers," "Introduction to the finite-difference time domain method," and "Technologies and applications of wave absorber II." $\mathrm{He}$ is a member of the IEEE, IEICE, IEEJ, JIEP, and AIJ. He has had more than 500 papers published in reviewed journals and international conferences. 